\title{
DESINCORPORACIÓN DEL SUELO COLECTIVO Y EL SISTEMA URBANO EN CHINA $1990-2015$
}

\author{
Liu Xuedong
}

Fecha de recepción: 19 de octubre de 2016. Fecha de aceptación: 13 de enero de 2017.

\begin{abstract}
RESUMEN
La urbanización es un proceso indispensable para lograr la modernización de una economía, y con ella se esperaría potencializar aún más el crecimiento a través de trasladar los factores productivos desde los sectores tradicionales con bajo nivel de productividad hasta los más dinámicos y, al mismo tiempo, concentrarlos paulatinamente en las zonas urbanas. En un país como China, donde existe una dominante dualidad en el sistema de la tenencia de tierra: por un lado, la que se localiza en las áreas rurales y es de propiedad colectiva que se destina a las actividades agrícolas; por otro lado, el suelo ubicado en las ciudades y es de propiedad estatal con fines urbanos.
\end{abstract}

Palabras clave: urbanización, tenencia de tierra, población urbana, ciudades, desincorporación del suelo colectivo.

Clasificación JEL: N95, O18, P25, R14, R58.

\section{The Dissolution of Community-Owned Lands and the Urban System IN ChInA 1990-2015}

\begin{abstract}
Urbanization is an essential process to modernize an economy, and it should, in turn, fuel growth even more by transferring productive factors from traditional sectors with low productivity to more dynamic sectors, while at the same time gradually concentrating them in urban zones. In countries like China, a dual land ownership system is predominant. On the one hand, land located in rural areas is generally collectively owned and set aside for agricultural activities, and on the other, land in the cities is the property of the state and allocated to urban purposes.
\end{abstract}

Key Words: Urbanization, land ownership, urban population, cities, dissolution of community-owned lands.

${ }^{1}$ Facultad de Estudios Superiores Aragón, Universidad Nacional Autónoma de México (UNAM), México. Correo electrónico: xdong64@hotmail.com 


\section{DÉMEMBREMENT DU SOL COLLECTIF ET LE SYSTÈME URBAIN EN CHINE 1990-2015 \\ Résumé}

L'urbanisation est un processus essentielle pour assurer la modernisation d'une économie, et avec elle, on s'attendrait la potentialisation de la croissance en transférant les facteurs de production des secteurs traditionnels à faible productivité aux plus dynamiques, et en même temps, les concentrer progressivement dans les zones urbaines. Dans un pays comme la Chine, où une dualité dominante existe dans le système de la propriété foncière que, d'un part, celle qui est localisé dans les zones rurales est de propriété collectif et est destiné aux activités agricoles ; d’autre part, le sol situé dans les villes à des fins urbaines est détenu par l'État.

Mots clés: urbanisation, propriété foncière, population urbaine, villes, démembrement du sol collectif.

\section{DESINCORPORAÇÁO DA TERRA COLETIVA E SISTEMA URBANO NA CHINA 1990-2015}

\section{Resumo}

A urbanização é um processo indispensável para conseguir a modernização de uma economia, e com ela esperar-se-ia potencializar ainda mais o crescimento através de transladar os fatores produtivos desde os setores tradicionais com baixo nível de produtividade até os mais dinâmicos e ao mesmo tempo os concentrar paulatinamente nas áreas urbanas. Num país como a China, onde há uma dualidade dominante no sistema de posse da terra, por um lado, a que está localizada em áreas rurais é de propriedade coletiva e é destinado para atividades agrícolas; por outro lado, o solo que está localizado nas cidades é de propriedade estatal com fins urbanos.

Palavras-chave: urbanizaçáo, posse da terra, população urbana, cidades, desincorporação de terras coletivas.

\section{0-2015年中国集体土地的消解及城镇制度}

刘学东

摘要

城市化是一个国家实现现代化必不可少的过程, 随着城市化进程, 我们期 待能够通过将生产因素由产能水平低的传统部门逐渐转移到城市来进一步 促进经济增长。在中国，土地所有制存在着二元结构：一方面，在农村， 土地是集体所有的, 用于农业活动; 另一方面, 城市土地是国有的, 用于 城市建设。

关键词：城市化 土地所有 城市人口城市 集体土地的消解 


\section{INTRODUCCIÓN}

La urbanización es un proceso indispensable para lograr la modernización de una economía, y con ella se esperaría potencializar aún más el crecimiento a través de trasladar los factores productivos desde los sectores tradicionales con bajo nivel de productividad hasta los más dinámicos y al mismo tiempo concentrarlos paulatinamente en las zonas urbanas. En un país como China, donde existe una dominante dualidad en el sistema de la tenencia de tierra: por un lado, la que se localiza en las áreas rurales y es de propiedad colectiva que se destina a las actividades agrícolas; por otro lado, el suelo ubicado en las ciudades y es de propiedad estatal con fines urbanos. Ante el proceso acelerado de urbanización y los ajustes pertinentes del crecimiento económico que se han llevado a cabo durante los últimos 25 años, la desincorporación del suelo de propiedad colectiva ha sido necesaria y ha desempeñado un papel crucial en la formación y evolución del sistema urbano.

Este trabajo está estructurado en cuatro secciones. La primera es una descripción breve sobre el panorama general del sistema urbano chino en la actualidad; en la segunda se analizarán los detalles relacionados con el proceso de urbanización efectuados en el lapso de 1990-2015 y sus respectivas características; en seguida se explicará porqué los procesos de urbanizar la población y el suelo se encuentra desfasados. Finalmente, se presentarán los retos que enfrentará el desarrollo del sistema urbano en el futuro.

\section{PANORAMA GENERAL DEL SISTEMA URBANO Y EL PROCESO DE URBANIZACIÓN EN LA ACTUALIDAD}

El modelo del crecimiento económico adoptado por China desde finales de los años setenta del siglo pasado, cuyo eje principal consiste en la aplicación de medidas orientadas a reformas económicas y de apertura comercial, ha arrojado resultados sobresalientes en el ámbito del crecimiento económico. Así, pues, desde 2010 la economía ha empezado a ubicarse como la segunda potencia mundial en términos del producto interno bruto (PIB). ${ }^{1}$

\footnotetext{
De acuerdo con las cifras publicadas por el Banco Mundial (вм), en términos del PIB la economía china se encontraba en la posición 11 en 1980, con un importe de 188.2 billones de dólares; en contraste con 2768.9 billones de dólares registrados por el mismo indicador de Estados Unidos. En 2011 las dos naciones arrojaron cifras de 7298.1 y 15094.0 billones de dólares, respectivamente. Esto es, en un lapso de 31 años, la brecha entre las dos economías se redujo de 14.9 a 2.1 .
} 
De la misma manera, el país asiático también ha logrado avances importantes en el proceso de urbanización y en la integración entre zonas rurales y urbanas con el fin de reducir las brechas del ingreso y del bienestar social entre campesinos y residentes urbanos. En "El Programa Nacional de la Nueva Urbanización, 2014-2020" ${ }^{2}$ publicado en marzo de 2014, se detalló la situación actual del sistema urbano y se fijó la meta de elevar el nivel de urbanización desde $52.6 \%$ en 2012 a aproximadamente $60 \%$ en $2020 .^{3}$

Con ello y junto con otras políticas pertinentes, se esperaría impulsar aún más el crecimiento económico y alcanzar la duplicación del ingreso disponible per cápita al finalizar la segunda década de presente siglo. De acuerdo con los argumentos, tanto teóricos como empíricos, el proceso de urbanización podría propiciar el crecimiento económico en los siguientes aspectos:

- Durante el proceso de industrialización, se registra movimiento masivo de los factores productivos, sobre todo la mano de obra desde el campo hasta las ciudades, y mediante esta reasignación de recursos desde los sectores primarios y tradicionales con bajo nivel de productividad laboral a los manufactureros y de servicios modernos con alto nivel de productividad, se generaría el aumento adicional de la eficiencia en las utilizaciones de los insumos respectivos.

- Por su parte, el auge y la concentración regional de los sectores manufactureros y de servicios modernos en las ciudades tanto ya existentes como las de nueva creación, propiciarían la economía de escala que potencializaría aún más el crecimiento económico.

- Asimismo, la concentración de las actividades económicas en las zonas urbanas, facilitaría la movilidad de todos los factores productivos debido a las infraestructuras y los servicios adecuadamente instalados, la acumulación de conocimientos y la capacitación de trabajadores, etcétera; todos ellos que generarían "la externalidad positiva" (Henderson, 2000, 2009; Fujita et al., 1999). de marzo de 2014, Beijing, China. población urbana y para contabilizarla se incluyen tanto los con registros urbanos como aquellos no urbanos pero que llevan más de seis meses consecutivos viviendo en las zonas clasificadas como urbanas. En 2013, estas áreas relacionaban con las 658 ciudades y 20113 Zhens (localidades administrativas al nivel más bajo dentro de la jerarquía del sistema chino en la administración pública que se ubica por debajo de los gobiernos central, provincial, distrital y municipal). 
- Políticas adecuadamente diseñadas e instrumentadas de urbanización podrían promover el uso más eficiente del suelo tanto en su asignación entre el sector rural y el urbano como dentro de la misma ciudad (Henderson, 2009).

Las cifras históricas más recientes confirmaron lo anterior, puesto que la población urbana al nivel mundial registró una tasa de crecimiento anual entre 5 y $6 \%$ en un lapso de 15-20 años, después de la Segunda Guerra Mundial para las economías del desarrollo en su fase de industrialización más acelerada. A consecuencia de lo anterior, las mismas naciones han sido paulatinamente transformadas desde sociedades con nivel de urbanización desde $20-25 \%$ hasta $70-85 \%$ (Henderson, 2009). En comparación con la velocidad registrada por las economías desarrolladas, el proceso de urbanización observado en los países emergentes ha sido más rápido. Precisamente por las contribuciones de ellos, la urbanización al nivel mundial se ha acelerado desde 1960, pues en un lapso de 43 años, la población urbana se triplicó al pasar de mil millones a 3.3 mil millones en 2003. De acuerdo con las proyecciones de Naciones Unidas, se espera la incorporación de otros mil millones en un lapso de 15 ańos futuros de 2003 a 2018 (Banco Mundial, World Development Report, 2009).

En su caso particular, la economía china ha mantenido un paso firme y sostenido con una tasa del crecimiento anual sobresaliente durante los últimos 30 años. Acompañado a este incremento, la urbanización también ha experimentado el proceso acelerado, pues de 1978 a 2015, la población urbana pasó de 170 millones a 771.2 millones de personas, localizadas tanto en las ciudades como en las localidades "semi urbanas (Zhen)", y el nivel de urbanización global aumentó de 17.9 a $56.1 \%,{ }^{4}$ con un incremento promedio anual

Es importante señalar que las cifras presentadas aquí sobre el nivel de urbanización aparentemente implican algunas deficiencias de comparación, pues mientras en 1978, la población urbana solamente se refería a la parte de residentes con registros urbanos; en 2013, la misma cifra no solamente incluía esa población particular, sino también aquellas personas que vivían en las ciudades por más de seis meses de manera consecutiva a pesar de ser miembros no pertenecientes a la categoría de residentes urbanos.

Para mayor detalle sobre el sistema en la administración de población en China, puede consultar: Xuedong, Liu (2014), "Régimen de tenencia de tierra y proceso de urbanización ante las recientes reformas agrarias. Estudios empíricos de China y México en la época moderna”, en Yolanda Trápaga Delfín (coord.), América Latina y El Caribe-China. Recursos Naturales y Medio Ambiente, primera edición, Red Académica de América Latina y el Caribe sobre China, Unión de Universidades de América Latina y el Caribe / Universidad Nacional Autónoma de México, México, 2013, pp. 175-194. 
de 1.03 puntos porcentuales. Además, el número de ciudades aumentó de 193 a 658, y el número de las "semiurbanas" lo hizo de 2173 a 20113 en los dos mismos ańos.

En el cuadro 1 se puede observar que, entre un total de 658 ciudades, seis se refieren a las conocidas como "megas" en 2010, con un número de población urbana superior a 10 millones de personas por unidad, en contraste con "nula localidad" en 1978. De igual manera, las con el número de habitantes entre 1 y 10 millones también subieron, al pasar de 29 a 134 para los dos años iguales. Por su parte, la distribución de población urbana entre los estratos distintos de las ciudades por tamaño indica que la mitad vive en los 20 mil centros "semiurbanos", 40\% se encuentra en las 652 ciudades con el número de habitantes menor a 10 millones, y casi $10 \%$ restante en las seis "megas" urbes.

Por distribuciones regionales, en 2010 el nivel de urbanización logrado en la parte Oriente fue $62.2 \%$, mientras que en las regiones Central y Occidente se ubicaba en 48.5 y $44.8 \%$, respectivamente. ${ }^{5}$ Además, en la región del Este se localizan los tres grandes círculos urbanos que se denominan como el cinturón económico de Beijing-Tianjin-Hebei, la Delta del Río Yangtze y la del Río Perla, todos ellos con nivel alto de urbanización, pues en suma concentran el 18\% de la población del país y, aportan el 36\% del pIB en una superficie de $2.8 \%$ del territorio nacional. Cabe mencionar que, dentro de estas tres zonas urbanas, se encuentran ubicadas cinco de las seis "megas" ciudades: Beijing, Tianjin, Shanghái, Guangzhou y Shenzhen. En suma, la población urbana total de estas cinco localidades superó a 78 millones que representaban más de $10 \%$ de la cifra total de China, con nivel de urbanización de 87.4, 82.6, 94.2, 85.4 y $100.0 \%$, respectivamente (la sexta ciudad de esta categoría es Chongqi que se ubica en el Suroeste de China, y que en 2014 su población total era de 30.2 millones, incluyendo todos sus municipios y el área principal, y el nivel de urbanización era de 60.9\%).

Es importante señalar que el proceso acelerado de urbanización registrado en China durante los últimos 25 años se ha visto reflejado tanto por el incremento significativo de la población urbana, como por la expansión masiva del área urbana. De acuerdo con las cifras arrojadas por el Anuario Estadístico de las Ciudades Chinas y el Reporte del Desarrollo de Zonas Urbanas Nuevas (Wang y Ma, 2016), solamente para el periodo de 2000-2010, la superficie urbana se expandió con una tasa acumulada de $83.4 \%$, en contraste con 
Cuadro 1. Distribución de las ciudades de acuerdo con el número de población ubana 1978-2010

\begin{tabular}{lrr}
\hline \multirow{2}{*}{ Tamaño de ciudades } & \multicolumn{2}{c}{ Número de ciudades } \\
\cline { 2 - 3 } & 1978 & 2010 \\
\hline$\geq 10$ millones & 0 & 6 \\
De 5 a 10 millones & 2 & 10 \\
De 3 a 5 millones & 2 & 21 \\
De 1 a 3 millones & 25 & 103 \\
De 0.5 a 1 millón & 35 & 138 \\
Menor 0.5 millones & 129 & 380 \\
Total & 198 & 658 \\
Zhens & 2173 & 19411 \\
\hline
\end{tabular}

Fuente: "VI Censo de Población". Oficina Nacional de Estadísticas de China.

45.9\% reportado por el aumento de población urbana. Esto implica que una parte importante del suelo de propiedad colectiva y destinada anteriormente al uso rural ha tenido que ser desincorporada de su régimen anterior y ha sido transformada como propiedad estatal para su posterior incorporación a la formación de área integral de las ciudades, destinándola a los usos urbanos respectivos, tales como las construcciones de viviendas, infraestructuras básicas, centros comerciales, parques recreativos, etcétera.

\section{CARACTERÍSTICAS DEL PROCESO DE URBANIZACIÓN EN CHINA, 1990-2015}

Durante los últimos 25 años, el proceso de urbanización registrado en China ha sido extraordinario en términos no sólo del volumen de población involucrada, sino también de la tasa del crecimiento, ya que se ha urbanizado un total de 474.6 millones de personas, con una tasa de crecimiento anual de $3.9 \%$ en promedio. Ante ello, es importante reconocer que este ritmo ha venido disminuyendo durante este lapso, al pasar de $4.5 \%$ en el primer decenio (1990-2000) a 3.9\% en el segundo decenio (2000-2010), para ubicarse en $2.9 \%$ durante los últimos cinco ańos (véase cuadro 2 ). A pesar del avance 
Cuadro 2. Tasa del crecimiento de población urbana y nivel de urbanización en China 1990-2015

\begin{tabular}{lccc}
\hline Años & $\begin{array}{c}\text { Población urbana }^{\star} \\
\text { (Millones de personas) }\end{array}$ & $\begin{array}{c}\text { Tasa de urbanización } \\
(\%)\end{array}$ & $\begin{array}{c}\text { Tasa de crecimiento } \\
\text { en promedio anual (\%) }\end{array}$ \\
\hline 1990 & 296.5 & 26.2 & $4.7^{* \star}$ \\
2000 & 458.8 & 36.9 & 4.5 \\
2010 & 670.0 & 50.3 & 3.9 \\
$2015^{\star \star \star}$ & 771.2 & 56.1 & 2.9 \\
\hline
\end{tabular}

Notas: *La población urbana es la suma de los residentes permanentes registrados en las ciudades y las localidades Zhen incluyendo los tanto con registros poblacionales urbanos como los rurales pero que estos ya llevan más de seis meses conseccutivos viviendo en la misma localidad ya sea ciudad o sea Zhen; ** Es la cifra registrada de 1978 a 1990 en promedio anual; *** Cifras de acuerdo con el Reporte Anual del Desarrollo Social Oficina Estatal de Estadísticas de China 2016.

Fuente: IV V y VI Censo Nacional de Población Oficina Estatal de Estadísticas de China.

significativo observado, en comparación con otras economías durante sus respectivos lapsos de urbanización acelerada, de un rango de 5-6\%, la cifra de China ha sido inferior.

Por lo tanto, con respecto a otras economías del mundo, el proceso de urbanización llevado a cabo en China se ha distinguido al menos en tres aspectos: la definición de población urbana y el consecuente cálculo del nivel de urbanización; la contribución significativa al proceso de urbanización por parte de las localidades semiurbanas, y la expansión excesiva de áreas urbanas.

\section{i. A pesar de los ajustes pertinentes para determinar la población urbana, se dista todavía en relación con los estándares internacionales aplicados en la actualidad.}

Para entenderlo mejor, es importante recordar que, como parte integral del sistema de planificación centralizada al nivel nacional, el patrón inicial en la administración poblacional se ha caracterizado por una evidente coexistencia de dos segmentos de registros: habitantes urbanos y rurales, de acuerdo a su lugar de residencia. Bajo este mecanismo, los habitantes con registros urbanos se encuentran con un nivel de vida y de bienestar social superior a los no urbanos, además de contar con una mejor cobertura de servicios sociales (Liu, 2014, 2015). Por su parte, para cada categoría la pertenencia se da por herencia y entre las dos se ha impuesto un control estricto tanto en el arraigo del 
lugar de residencia como en la modificación de categoría, sobre todo para los catalogados en el segmento rural. Para el fin de medir el nivel de urbanización, se calculaba de acuerdo con la participación de los habitantes con registros urbanos dentro de la población total, independientemente de su lugar de residencia y de actividades económicas dedicadas por ellos.

De esta forma, las reformas económicas y, en consecuencia, la industrialización y la urbanización, que se han llevado a cabo respectivamente desde hace tres décadas, han permitido que las actividades no agrícolas hayan rebasado su delimitación exclusiva en las zonas urbanas y se hayan extendido a las zonas rurales. Al mismo tiempo los campesinos no solamente trabajan en la tierra cultivando los distintos productos agropecuarios, sino también realizan actividades no agrícolas por iniciativa propia, ya sea en su comunidad, o en las ciudades, con lo cual las asignaciones de recursos productivos se están aproximando a la necesidad de lograr la eficiencia óptima en sus usos respectivos. ${ }^{6}$

Con el transcurso del tiempo, especialmente ante las reubicaciones de los recursos productivos entre sectores económicos y entre localidades, todas derivadas de la aplicación de las medidas de reformas económicas y de apertura comercial, la forma convencional de computar el nivel de urbanización ha arrojado cada vez más que antes las deficiencias.

En primer lugar, la aplicación paulatina de medidas de reformas económicas ha flexibilizado las restricciones impuestas a la movilidad de los campesinos y, por lo tanto, las personas con registros rurales pueden vivir y trabajar en las ciudades dedicándose a las actividades económicas que no tienen nada que ver con el sector agrícola. Sin embargo, ellos no son habitantes urbanos por registro y no están incluidos en las cifras oficiales para contabilizar el avance de urbanización, por lo tanto, los datos estadísticos referentes no reflejarían la realidad ni tampoco serían comparables con los registrados al nivel mundial.

En segundo lugar, en los años noventa, con el desmantelamiento del sistema de comuna popular (la última jerarquía del gobierno del sistema político chino anterior, desde arriba hacia abajo después del gobierno central, pro-

6

Esto es, las comunidades rurales están consideradas como un espacio que involucra actividades agrícolas, pero no exclusivas, por lo tanto, es necesario arribar a una nueva conceptualización del desarrollo rural que considere el carácter heterogéneo y complejo del espacio rural y las cambiantes condiciones del campo en el marco de la mundialización de las economías y la creciente presencia de flujos internacionales de mercancías y personas.

Roberto Escalante Sernerena y Fernando Rello Espinosa (2000), "El sector agropecuario mexicano: los desafíos del futuro”, Comercio Exterior, noviembre, México, pp. 984-987. 
vincial, distrital y contado), se han establecido dos tipos de organizaciones locales: Xiang y Zhen para suplirlo.

Usualmente las localidades Xiang se consideran como áreas rurales y las Zhen como zonas urbanas, pues en estas últimas localidades generalmente el nivel de industrialización ha sido alto y las actividades agrícolas ya no son fuentes principales del ingreso de los habitantes. Asimismo, la proliferación de las actividades no agrícolas y de la formación de empresas en las zonas rurales (Town México y Village Enterprises, TVE, por sus siglas en inglés) desde el principio de los años ochenta hasta al menos la mitad de los noventa ha contribuido de manera importante al crecimiento económico, sobre todo en el aspecto de fomentar el desarrollo de las zonas rurales catalogadas como Zhen. Durante ese lapso, las TVE generaron más de 100 millones de empleos, los cuales impulsaron aún más el crecimiento de las Zhen en términos no solamente de su consolidación sino también de su nueva creación.

A pesar de que esta clasificación inicial entre Xiang y Zhen con frecuencia ha sido arbitral, lo ocurrido posterior en las dos zonas distintas, sobre todo el impulso logrado en el crecimiento económico en los sectores no agrícolas ha justificado tales clasificaciones en la mayoría de los casos. En este término, la inclusión de habitantes localizados en las Zhens como población urbana, sin duda, ya es una necesidad urgente para medir adecuadamente el nivel real de urbanización. Además, si se considera que en la actualidad el número de habitantes en las Zhens se sitúa en el alrededor de 20 mil personas por localidad, se podría inferir implícitamente el límite para distinguir zonas urbanas y rurales. Por ello, el criterio para calcular el nivel de urbanización actualmente en China que incluye los residentes permanentes en las Zhens se podría tomar como un paso importante para acercar a los utilizados al nivel mundial.

En tercer lugar, bajo este nuevo contexto, los campesinos pueden seleccionar libremente entre trabajar en su propia comunidad dedicados a las actividades, tanto agrícolas como las no agrícolas, y/o emigrar del campo a las ciudades dejando totalmente las actividades primarias. De cierta manera, el sistema original en la tenencia del suelo ha empezado a desmantelarse, al perder la vinculación estricta entre los campesinos, las actividades agrícolas y la tierra de propiedad colectiva. Sin embargo, el libre movimiento de los campesinos desde el campo hasta las ciudades no se ha acompañado con la reforma pertinente en la administración poblacional al seguir manteniéndose dentro del patrón de registro de habitantes rurales a pesar de su evidente residente urbano. De acuerdo con los reglamentos vigentes, ellos todavía se encuentran imposibilitados en acceder a los servicios básicos proporcionados por las autoridades locales de las ciudades. 
Así pues, los campesinos inmigrantes en las ciudades con frecuencia tienen que superar varias dificultades para permanecerse ante las condiciones y los tratos desiguales que los habitantes registrados como residentes urbanos. Concretamente, no solamente ellos tienen que conformarse con el nivel relativamente bajo de salarios y de otras prestaciones laborales, sino también no les queda más que tolerar al acceso restringido de los servicios básicos. El ejemplo evidente se relaciona con las restricciones impuestas a sus hijos al momento de recibir la educación tanto básica como media y superior. Con respecto al primer caso, los campesinos emigrados se ven obligados a pagar una cantidad monetaria adicional, como una compensación al servicio recibido. En el segundo caso, con el propósito de realizar el examen del ingreso a las universidades y recibir la educación superior al terminar la preparatoria, estos jóvenes tienen que trasladarse a su lugar de origen en lugar de a las ciudades donde han vivido con sus padres y han hecho estudios de primaria, secundaria y preparatoria.

De lo anterior, se aprecia que los avances efectuados hasta la fecha en materia de llevar a cabo el proceso de urbanización todavía tienen varios pendientes por atenderse, especialmente en el aspecto de igualar los servicios básicos para toda la población urbana tanto los registrados dentro del segmento urbano como los del rural. Cómo mejorar las condiciones de vida de los campesinos emigrados en las ciudades, especialmente cómo hacer llegar los mismos servicios básicos a estos nuevos residentes que los ya existentes, es un gran reto para las autoridades en todos sus niveles (Liu et al., 2015).

Por lo tanto, las cifras estadísticas reportadas en la actualidad definitivamente no han reflejado la realidad por la parcialidad y la diferenciación registradas en el proceso de urbanización. Además, la definición sobre la población urbana todavía no ha sido compatible con los criterios utilizados en la mayoría de otros países del mundo.

\footnotetext{
$\mathrm{Al}$ respecto es importante mencionar que en las grandes ciudades, por ejemplo, Beijing, Shanghái, Tianjin, etcétera, además de ser lugares atractivos para los campesinos emigrados, generalmente también concentran las mejores universidades e instituciones de educación superior, y ofrecen mayor número de plazas para los aspirantes a recibir educaciones superiores en comparación con otras entidades provinciales. Por ello, participar en el examen del ingreso a la educación superior en estas ciudades significa una ventaja de tener una posibilidad más alta de ser aceptados que en su lugar de origen. En casos extremos, la diferencia de las calificaciones para los aspirantes entre las distintas entidades provinciales (incluyendo las provincias, las zonas autónomas y las ciudades directamente sujetas a la administración central) puede llegar hasta más de 100 puntos en un total de 700 en promedio, en el acceso a la misma facultad de una universidad en particular.
} 
ii. Dentro de la población urbana se incluyen dos diferentes residentes: los registrados en las ciudades y los de las localidades Zhen, y estas uiltimas entidades geográfcas han contribuido de manera sobresaliente al avance de urbanización en el lapso analizado.

En 2010, dentro de un total de 670.0 millones habitantes urbanos registrados (véase cuadro 2), 39.7\%, o 266.2 millones se encuentran ubicados en las localidades Zhen. En comparación con las cifras arrojadas en 1990, de 40.4\%, la estructura se mantiene casi sin variación, esto es, de cada 10 residentes urbanos, cuatro viven en las casi 20 mil localidades llamadas de Zhen y, seis en las 658 ciudades.

Para los dos años analizados, se puede observar que al menos las tres principales ciudades con índices mayores a los 10 millones de habitantes (Beijing, Shanghái y Tianjin solamente, ya que otras tres son de recién creación o del aumento de categoría, y por eso no se puede realizar la comparación similar) han perdido su importancia dentro del total de la población urbana, al pasar de 11.1 a $10.1 \%$; durante los últimos cinco años esta tendencia no se ha modificado (véase cuadro 3). De acuerdo con cifras de 2014 (con excepción de Tianjin, la cifra es de 2015) la población urbana en estas tres zonas urbanas sumaba un total de 56.1 millones de habitantes, los cuales implican una participación de $7.3 \%$ dentro del total, o una reducción neta de 2.8 puntos porcentuales.

En otras palabras, a pesar del crecimiento significativo de la población en las "megas" ciudades durante las últimas dos décadas, su ritmo ha sido inferior en comparación con el registrado al nivel nacional. Lo anterior significaría que, en primer lugar, las 652 ciudades restantes han aportado con mayor contribución al proceso de urbanización, ganando 1.7 puntos porcentuales netos en el lapso de 1990-2010. En segundo lugar, las otras tres urbes con población urbana superior a 10 millones de personas también han desempeñado un papel importante en la absorción de la población recién urbanizada, pues su importancia pasó de 2.5 a 5.2\% de 2010 a 2014 . Tal como se refleja en el cuadro 3, la población urbana en las seis "megas" ciudades en 2014 sumaba 96.5 millones, que representaban una participación de $12.5 \%$ dentro del total; las 652 ciudades restantes con $47.8 \%$; y las de Zhen con 39.7\%. 
Cuadro 3. Población urbana y tasa de urbanización en las mega ciudades 2014

\begin{tabular}{lccc}
\hline Localidades & $\begin{array}{c}\text { Población total } \\
\text { (Millones de personas) }\end{array}$ & $\begin{array}{c}\text { Población urbana } \\
\text { (Millones de personas) }\end{array}$ & $\begin{array}{c}\text { Tasa de urbanización } \\
\text { (\%) }\end{array}$ \\
\hline A nivel nacional & 1374.7 & 771.2 & 56.1 \\
Beiïng & 21.5 & 18.8 & 87.4 \\
Shanghái & 24.3 & 22.9 & 94.2 \\
Tianjin (2015) & 15.5 & 14.4 & 82.6 \\
Guangzhou & 13.1 & 11.2 & 85.4 \\
Shenzhen & 10.8 & 10.8 & 100.0 \\
Chongqi & 30.2 & 18.4 & 60.9 \\
Suma de seis ciudades & 115.4 & 96.5 & 83.6 \\
\hline
\end{tabular}

Fuente: Oficina estatal de estadísticas de China y las oficinas locales de cada ciudad.

iii. Otra caracteristica en este proceso de urbanización ha sido la eminente superioridad de la expansión territorial en las manchas urbanas en comparación con el crecimiento de la población urbana.

De acuerdo con cifras de distintas fuentes, se ha observado que la velocidad en el aumento de superficie urbanizada ha sido entre 1.5 y 2.3 veces mayor a la de población. Por un lado, en el periodo de 1981-2007, el área urbanizada ha crecido con una tasa de $6.2 \%$ en promedio anual, mientras que los residentes urbanos han hecho con $4.2 \%$, con una diferencia de 2.0 puntos porcentuales entre ambas; de 2000 a 2007 la brecha se amplía más todavía con 3.1 puntos porcentuales al arrojar 6.8 y 3.7\%, respectivamente (Cai, 2013). Es decir, la expansión de zonas urbanas se ha acelerado a partir de 2000. Por otro lado, las cifras reveladas por la Oficina Nacional de Estadísticas de China demuestran que de 1989 a 1996, las ciudades han extendido con un total de 1649 kilómetros cuadrados, al pasar de 3270 a 4 910, con una tasa de crecimiento acumulado de $50.2 \%$; y durante el mismo lapso, la población urbana fue de 21.9\%; por ello, los dos procesos de urbanización registraron una diferencia de 2.3 veces. Para el periodo de 2000-2012, la brecha entre las dos variables involucradas ha reducido para ubicarse en 1.8 veces, no obstante, sigue siendo importante. 
De acuerdo con lo observado al nivel mundial, lo ocurrido en China parecería una contradicción, ya que se trata de una economía en desarrollo. Para esta fase, la urbanización poblacional generalmente es acelerada y propiciada por la llegada masiva de campesinos a las ciudades; en contraste, la expansión de zonas urbanas registra avances de menor magnitud. Sin embargo, cuando la urbanización poblacional una vez alcanza un nivel en el alrededor de $80 \%$, usualmente se encuentra en un estado estacionario considerando el término de la migración del campo a ciudad para las economías desarrolladas. Por su parte, la ampliación de zonas urbanizadas podría prolongarse por más tiempo todavía, e inclusive podría ser considerada como un proceso constante, ya que las demandas para mejorar las condiciones de vivienda con más espacio y mayor número de unidades, ampliar los espacios recreativos, comerciales y de áreas verdes, etcétera, aumentan de acuerdo con el crecimiento económico y el desarrollo social.

Las cifras registradas por el crecimiento tanto de la población urbana como de las áreas urbanas a nivel mundial han confirmado lo anterior. Por un lado, los cálculos realizados por Cai (2013: 310) indican que de 1955 a 2005, las áreas incorporadas en las ciudades se duplicaron para los países miembros de Organización para la Cooperación y Desarrollo Económicos (OCDE), mientras que otros del resto del mundo lo hicieron con 4.2 veces $(2.9 \%$ en promedio anual). Por otro lado, durante un lapso de 43 años, la población urbana en los países industrializados registró una tasa de crecimiento mucho menos dinámico que el resto del mundo, y prácticamente se mantuvo sin cambios (Banco Mundial, 2009). De tal manera, se puede inferir que la incorporación de nuevas áreas en la mancha urbana ha arrojado una magnitud de menor intensidad que la de población urbana para los países en desarrollo.

Por el contrario a lo registrado al nivel mundial, el exceso en el aumento de áreas urbanizadas se podría explicar por dos factores: la política diseñada e instrumentada poco eficiente en la incorporación del suelo al proceso de urbanización, y los impedimentos impuestos por las autoridades de distintos niveles para urbanizar la población, tal como se detallará a continuación.

\section{PROCESOS DISTINTOS PARA URBANIZAR EL SUELO Y LA POBLACIÓN EN CHINA}

Antes de la instrumentación de políticas de promover la apertura comercial y de reformas económicas en China, el proceso de urbanización tanto del suelo como de la población se encontraba estrictamente controlado por las autoridades sujeto a los requerimientos de planificación centralizada. Bajo este 
contexto, existía un sistema dual en varios aspectos: el campo vs. las ciudades; la tierra urbana $v$ s. la rural; los residentes rurales $v s$. los urbanos; la producción agrícola $v$ s. las actividades secundarias y terciarias, etcétera. De tal manera, la combinación de estos elementos forma dos círculos prácticamente cerrados: en las áreas rurales los residentes están catalogados como campesinos y con registro de población rural, que trabajan exclusivamente en el sector agrícola; en contraste, en las ciudades, los habitantes están registrados como población urbana que se dedican a las actividades secundarias y terciarias. Cabe mencionar que entre estos dos conjuntos se tenían barreras que impedían pasar libremente a la población involucrada de una área a otra, sobre todo para los campesinos (de transformar los residentes con registros rurales a urbanos).

Con el avance de implementar las medidas encaminadas a promover la eficiencia en la asignación de los factores productivos, los impedimentos impuestos se han flexibilizado paulatinamente. Por ejemplo, los campesinos pueden escoger libremente trabajar en el sector agrícola o en otras actividades económicas tanto en su lugar de origen o fuera de ello. Sin embrago, en el ámbito relacionado con el cambio de usos del suelo, las restricciones todavía no han sido modificadas; esto es, el único mecanismo sigue siendo la expropiación estatal para transformar la tierra rural a usos urbanos. Así pues, cualquiera desincorporación el suelo rural de propiedad colectiva y su posterior incorporación a los usos urbanos tienen que obtener la autorización previa de la Secretaría de Recursos de Territorio Nacional, instancia del gobierno central para determinar anualmente el monto de desincorporación de tierra colectiva para cada entidad que involucra a las 23 provincias, cinco regiones autónomas y cuatro municipios, con un total de 32; y posteriormente, de acuerdo con el cupo determinado, las autoridades locales realizan las operaciones directamente con los campesinos.

Si bien durante el lapso de urbanización lenta, la necesidad de desincorporar suelo de propiedad colectiva era de poca cantidad, el monto de indemnización derivada de la expropiación también ha sido de poca consideración tanto para los campesinos como para las autoridades a sus distintos niveles. No obstante, este importe ha crecido significativamente ante el proceso acelerado de urbanización desde los años noventa, aparentemente debido a dos factores principales. Por un lado, los campesinos han empezado a exigir cantidades cada vez mayores que antes por concepto de pago de indemnización al desincorporar su tierra de propiedad colectiva. Por otro lado, la expansión de los centros urbanos y el surgimiento de los recién creados requieren la desincorporación de suelo de los campesinos con una magnitud cada vez más intensa que antes. Ante ello, las entidades locales con frecuencia se encuentran 
en dificultades para contar con suficientes recursos financieros propios para cubrir las necesidades para realizar pagos tanto en forma como en tiempo a los productores agrícolas.

Como siempre ha sucedido, que el mismo suelo expropiado para destinarlo a usos urbanos registra variaciones importantes en su valor en comparación con su destino original, y por ello este proceso de expropiación y subasta de tierra también es un proceso de distribuir este valor adicional derivado del cambio en los usos del suelo entre las autoridades, los desarrolladores y los campesinos. Además, debido al aumento significativo de los recursos financieros para cubrir las necesidades, las autoridades han optado a acudir a las instituciones crediticias y contraer endeudamientos para complementar la cantidad requerida de financiamiento y efectuar el pago por concepto de las indemnizaciones a los agricultores con propósito de obtener el suelo de propiedad colectiva transformándolo a la estatal; posteriormente, las mismas instancias gubernamentales subastan la tierra expropiada y de propiedad estatal a los desarrolladores inmobiliarios para recuperar los recursos financieros y cerrar el círculo.

De acuerdo con las estimaciones, el ingreso generado por el cambio en los usos del suelo se ha distribuido de la siguiente manera: las autoridades locales con 20-30\%; las empresas desarrolladoras con 40-50\%; el gobierno del pueblo con 25-30\%; finalmente, los miembros de la localidad con 5-10\%. En otras palabras, aproximadamente el $75 \%$ del valor derivado del cambio en el uso del suelo se ha dirigido como ingreso de las autoridades locales y de las empresas involucradas en este proceso, incluyendo los desarrolladores inmobiliarios y los bancos (Ma, 2013). La forma en la distribución del ingreso por este concepto ha propiciado que las autoridades locales se encontraran atrapadas y especialmente ansiosas de conseguir el cupón de expropiación del suelo colectivo con propósito de mejorar los ingresos del gobierno local, e inclusive para otros fines (corrupción, enriquecimiento ilícito, etcétera). Todo lo anterior ha motivado que la urbanización del suelo en las ciudades se ha convertido en un proceso auge y excesivo, sobre todo a partir de 2000 cuando el sector inmobiliario y de construcción ha crecido significativamente para satisfacer la demanda creciente de vivienda.

Con propósito de entender lo anterior, se presenta un caso práctico en litigio con Número 00374, dentro del juicio civil archivado en la Suprema Corte Judicial de la Provincia Jiangsu (Asesoría legal, 5 de junio de 2015).

En este caso, se encuentra involucrada una superficie con un total de $6000.5 \mathrm{~m}^{2}$, pertenecientes a dos pueblos. En su momento, la Oficina de Tierra Estatal del municipio Binghai y la Compaña del Desarrollo Inmobiliario 
Xin Fuda de Yancheng acordaron un convenio de compra-venta del lote mencionado con número $2011 \mathrm{G} 10$, a través de subasta pública, con un valor total de 8520710 yuans, lo cual se distribuyó a la siguiente manera de acuerdo con el cuadro 4:

Cuadro 4. Distribución de ingreso derivado del cambio en el uso del suelo

\begin{tabular}{lcc}
\hline Instancia receptora & Monto (Yuan) & Estructura (\%) \\
\hline Oficina de Tierra Estatal del municipio Binghai: & 7801030 & 91.6 \\
Gobierno local de Caiqian Zhen & 301080 & 3.5 \\
Habitantes y campesinos & 418600 & 4.9 \\
Total & 8520710 & 100.0 \\
\hline
\end{tabular}

Fuente: Li Yi (2015), "La Suprema Corte Judicial de la Provincia Jiangsu: Se cancela el convenio de la compra-venta del suelo que se encuentra en litigio legal", Asesoría legal 5 de junio de 2015. Recuperado de $<$ http://www.ask64.com/Html/3261.html>

\section{RETOS FUTUROS PARA LA URBANIZACIÓN Y EL DESARROLLO DEL SISTEMA URBANO EN CHINA}

La urbanización es un proceso que involucra varios actores de la economía y tiene impactos en aspectos distintos, por ello el avance equilibrado y con distribución del ingreso equitativo y justo entre ellos es de vital importancia para llevar a cabo el proceso de urbanización y del desarrollo del sistema urbano de manera óptima y sana. Ante las diferencias de propiedades del suelo entre los usos rurales y los urbanos y sobre todo las incompatibilidades existentes entre ellos dos, los problemas generados por el cambio de sus usos respectivos han propiciado desafíos importantes que podrían afectar el desarrollo normal de este proceso. Por ello, se requieren nuevas medidas para adecuar los sistemas actuales en la administración tanto del suelo como de la población.

\section{a) El sistema dual en la administración del suelo y de población y sus reformas pendientes}

Durante las últimas seis décadas el proceso de urbanización en China pasó de una etapa de lento crecimiento a la actual de aceleración; sin embargo, los mecanismos en la incorporación de suelo y de población a la formación del 
sistema urbano no han sido modificados, pues el patrón dual en la administración del suelo y de población hasta la fecha prácticamente se ha mantenido.

$\mathrm{Al}$ seguir el mecanismo actual de expropiar el suelo de propiedad colectiva para destinarlo posteriormente a los usos urbanos, el costo por las indemnizaciones ha registrado tendencia creciente ante las exigencias de los campesinos y de la mayor madurez del mercado de bienes raíces tanto en las ciudades como en las zonas rurales, sobre todo durante los últimos 10 años. Con frecuencia estas operaciones han propiciado serios enfrentamientos entre las autoridades municipales y los miembros de la comunidad agrícola, los cuales inclusive han creado conflictos profundos impactando negativamente en la estabilidad social. En este sentido, los costos crecientes tanto económicos como sociales por la aplicación de este modelo de urbanización han amenazado su factibilidad, ${ }^{8}$ y por ello, se requiere nuevas medidas pertinentes para flexibilizar los reglamentos vigentes para que el suelo de propiedad colectiva pudiera contar con mecanismos adecuados para su incorporación a los fines urbanos.

\section{b) Urbanización poblacional integral y completa es una tarea urgente de resolver}

De acuerdo con las cifras estimadas, de un total de más de 700 millones habitantes urbanos registrados en la actualidad, solamente 500 millones son residentes con registros urbanos que representan aproximadamente $70 \%$; y el $30 \%$ restante se clasifica como habitantes urbanos permanentes cuyos registros todavía se encuentran inscritos en el patrón de población rural. Además, durante los próximos 20 ańos, se espera que se incorporaran otros 200 millo-

La tierra colectiva es la propiedad de los pueblos que forma por tres partes: el suelo para uso agrícola; para construcción; y el del uso común, básicamente referido a las áreas dominadas por montańas, agua, pasto, etcétera. Por su parte, el suelo para construcción también está clasificado por tres categorías: la superficie dedicada a asentamientos humanos (solar), la parte ocupada para las infraestructuras del pueblo de interés común y la tercera para uso industrial y comercial. Es importante recalcar que ninguna parte de la tierra colectiva puede ser utilizada directamente para la construcción de viviendas destinadas a la venta a los residentes urbanos o a la población afuera de su comunidad. Además, cualquier modificación en el uso del suelo colectivo tiene que ser autorizada y transferida a la propiedad estatal por las autoridades locales de acuerdo con la planeación del gobierno central y, posteriormente, ser destinada a los usos tanto urbanos u otros para el interés público.

Con relación a las especificaciones impuestas al uso de tierra colectiva, se pueden consultar con la Constitución Artículo 10 (1982), la Ley de Tierra (1986), y la Ley de Administración de Tierra Colectiva (2003). 
nes de campesinos como población urbana en las ciudades; y de tal manera, el nivel de urbanización se ubicaría en el alrededor de $70 \%$ en 2030 . Ante la necesidad de igualar los servicios básicos proporcionados por las autoridades entre los residentes urbanos nuevos y los ya existentes, se estima una inversión con un monto total en el alrededor de 51 billones de yuanes ( 8 billones de dólares norteamericanos aproximadamente); esto es, 130.1 miles de yuans (20 miles de dólares de acuerdo con el tipo de cambio actual) para cada inmigrante. Alcanzar este fin, se podría implicar la urbanización integral y completa para toda la población urbana, independientemente del patrón del registro poblacional al que pertenecen; esto es, garantizar el suministro eficiente de los servicios relacionados con la educación, el seguro social y la salud, la vivienda, el mantenimiento de las infraestructuras, entre otros, ${ }^{9}$ durante los próximos 20 años. Bajo este escenario, la urbanización de los campesinos inmigrantes en las ciudades implicaría el goce pleno de los derechos y los tratamientos como residentes urbanos sin afectar el nivel alcanzado por los ya existentes, lo cual contribuiría a la eliminación completa de las barreras construidas desde hace más de 60 años entre las dos categorías de población.

\section{BIBLIOGRAFÍA}

Banco Mundial (вм) (2009), World Development Report, 2009, "Reshaping Economic Geography”.

Cai, F. (2013), "Opciones para avanzar el proceso de urbanización en China y las experiencias internacionales", en Fang Cai, El crecimiento económico chino desde enfoque de población, colección de obras propias del autor, Editorial de Ciencias Sociales de China, Beijing, China, pp. 300-316.

Consejo Estatal de China (2014), "El Programa Nacional de la Nueva Urbanización, 2014-2020", Publicaciones Oficiales del Consejo Estatal de China, núm. 9 de 2014, núm. de volumen 1476, publicado en 30 de marzo de 2014, Beijing, China.

Escalante Sernerena, R. y F. Rello Espinosa (2000), "El sector agropecuario mexicano: los desafíos del futuro", Comercio Exterior, noviembre, México, pp. 984-987. celebrada del 12 al 13 de diciembre de 2013 en Beijing, China. 
Fujita, M., P. Krugman y A. Venables (1999), “The Spatial Economy”, Cambridge, MIT Press.

Henderson J., Vernon (2000), "The Effects of Urban Concentration on Economic Growth", NBER Working Paper 7503. Recuperado de <http:// www.nber.org/papers/w7503>

(2009), "Urbanization in China, Policy Issues and Options, China Economic Research and Advisory Programme", Brown University and NBER, 14 de noviembre.

Li, Y. (2015), "En la Suprema Corte Judicial de la Provincia Jiangsu: Se cancela el convenio de la compra-venta del suelo que se encuentra en litigio legal", Asesoría legal, 5 de junio de 2015. Recuperado de <http://www. ask64.com/Html/3261.html>

Liu, X. (2014), "Soluciones legales para las viviendas de Xiao Chanquan", Finance de IFeng, 7 de julio, recuperado de <http://finance.ifeng.com/a/ 20140707/12665893_0.shtml>

(2014), "Régimen de tenencia de tierra y proceso de urbanización ante las recientes reformas agrarias. Estudios empíricos de China y México en la época moderna”, en Yolanda Trápaga Delfín (coord.), América Latina y El Caribe-China, Recursos Naturales y Medio Ambiente, 1era edición, 2013, Red Académica de América Latina y el Caribe sobre China, Unión de Universidades de América Latina y el Caribe, y Universidad Nacional Autónoma de México, México, pp. 175-194,

Liu, X., Q. Zhang, F. Chen, W. Bo, H. An e Y. Yan (2015), "La reestructuración económica y las nuevas estrategias del desarrollo en China", Cuaderno del Trabajo, Revista del Centro de Estudios China-México, Facultad de Economía, Universidad Nacional Autónoma de México, núm. 1, pp. 3-32.

Ma, Zh. (2013), "Dependencia excesiva del ingreso derivado del cambio en el uso del suelo para las finanzas del gobierno local elevaría el costo final de las viviendas", 27 de junio. Recuperado de <http://finance.sina.com.cn/zl/ china/20130627/174215941315.shtml>

"Programa Nacional de la Nueva Urbanización”, versión en discusión por la Reunión Central sobre Trabajos de Urbanización celebrada de 12 a 13 de diciembre de 2013 en Beijing, China.

Wang, Le y Ma Xiaoqing (2016), "El auge de crear nuevas zonas urbanas en China: una ciudad se complementa por 19 más a través de expansión anárquica (中国遍地造城热：一个市规划 19 个新城)”, 15 de julio de 2016, recuperado de <http:// data .163. com / 16/0715/07 /BS0FR2U100014MTN.html> 